\title{
Miniscrews: protocol and mechanics for treatment of Class II malocclusions
}

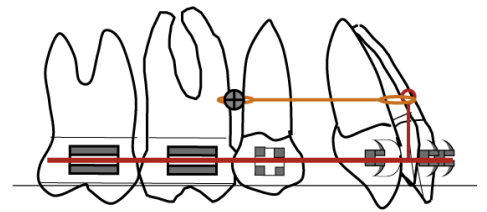

Direct mechanics

\section{Jacques FAURE, Arlette OUEISS}

\begin{abstract}
Miniscrews are the most important recent innovation in orthodontics and have won a place, we believe, in the routine appliance design of everyday orthodontic practice. They are, we think primarily applicable in the treatment of the most prominent feature of the malocclusions orthodontists regularly confront, an antero-posterior discrepancy. Accordingly we discuss the protocol for full banded and bonded Class II mechanotherapy with miniscrew anchorage for:
\end{abstract}

- Global arch or anterior sectional displacement;

- Direct or indirect mechanics;

- Sliding or loop mechanics.

We then demonstrate clinical procedures: placement of miniscrews, static and mobile sectors, strengthening sectorial anchorage, active forces, and space management.

Next we analyze the mechanics of treating complex cases with vertical as well as antero-posterior problems.

Throughout the article we illustrate the text with sketches and clinical records.

\section{KEYWORDS}

Mini-implants

Class // therapy

Protocol for use of mini-implants.

Conflict of interest: none Received: 11-2009. Accepted: 01-2010.

\section{1 - INTRODUCTION}

Miniscrews are no longer just a tool of last resort to be reserved for a few exceptional cases involving orthodontic preparation for extensive prosthetic rehabilitation, multiple loss of teeth, and asymmetrical tilting of the

Address for correspondence:

J. FAURE,

10 place Lannes,

32000 Auch,

faure_ja@club-internet.fr

A. OUEISS

aoueiss@hotmail.com 
occlusal plane but have become a basic component of our therapeutic arsenal.

Considerations confirming this conception include:

- Malocclusions are essentially antero-posterior discrepancies;

- the classification of malocclusion is based on antero-posterior relationships;

- orthodontic therapies, orthodontic mechanics, orthodontic objectives, and the results of orthodontic treatment are above all conceived in antero-posterior terms;

- an orthodontist is, accordingly, "Dr. Antero-Posterior".

The major application for miniscrews is, accordingly, in antero-posterior mechanotherapy. It seemed to us, therefore, that it would be a fruitful enterprise to state precisely the protocols for their employment in regularly referring to the most frequently encountered cases in our daily practices, Class II malocclusions. We shall present the clinical modalities followed by an analysis of the mechanics employed in the management of associated vertical problems.

We based our clinical choices on the anatomic date published in the literature ${ }^{2,3,9}$, the anchorage values of teeth and groups of teeth as calculated from scanner generated data $^{6,7,8}$, our theoretical analyses of full-banded and bonded mechanics ${ }^{1}$, the available material, and the primary papers describing the use of miniscrews in therapy ${ }^{4,5,10}$.

\section{1 - PROTOCOLS BASED ON SEVERITY OF THE DISORDER}

\section{2 - 1 - General therapeutic objectives, protocol and mechanics}

The objective defined a priori in the treatment plan strongly affects the choice mechanics to be used. For example, the occlusal situation may require (fig. 1):

- either movement only of a segment of teeth, the upper anteriors, that are in a Class II position,

- or a distal movement of all the upper teeth, in a Class II, division 1 case, distalization, for example, of the buccal and posterior teeth as well a major distal movement of the maxillary anteriors.

\section{2 - 1 - 1 - Sectional movement}

This is an optimal situation for orthodontists whose primary difficulty will be not to cause a loss anchorage in the upper arch, which would put the buccal teeth in a Class II relationship, thus complicating the original situation. Accordingly, they must place the least possible strain on the maxillary anchorage units.

To insure that they succeed, miniscrews can assist greatly, in two possible ways, through:

- indirect mechanics where the miniscrew reinforces the basic anchorage units of premolars and molars that are the stable unit from which 


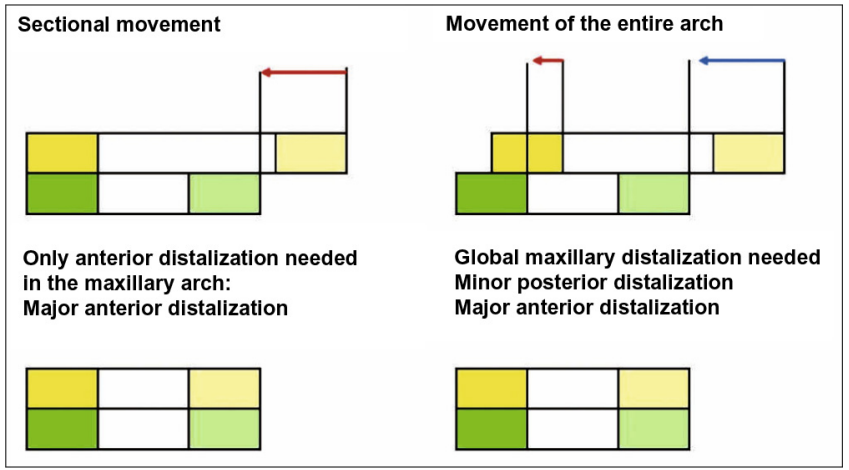

Figure 1

Sketch of the protocol designation according to severity of discrepancy (sketch describes occlusal relations of right hemi-arches).

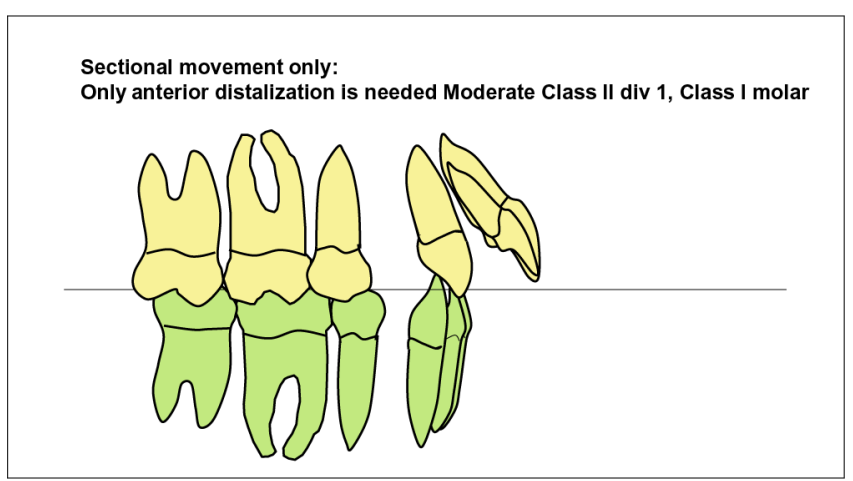

Figure 2

Sectional distalization only: buccal teeth that are in class I need no distalization.
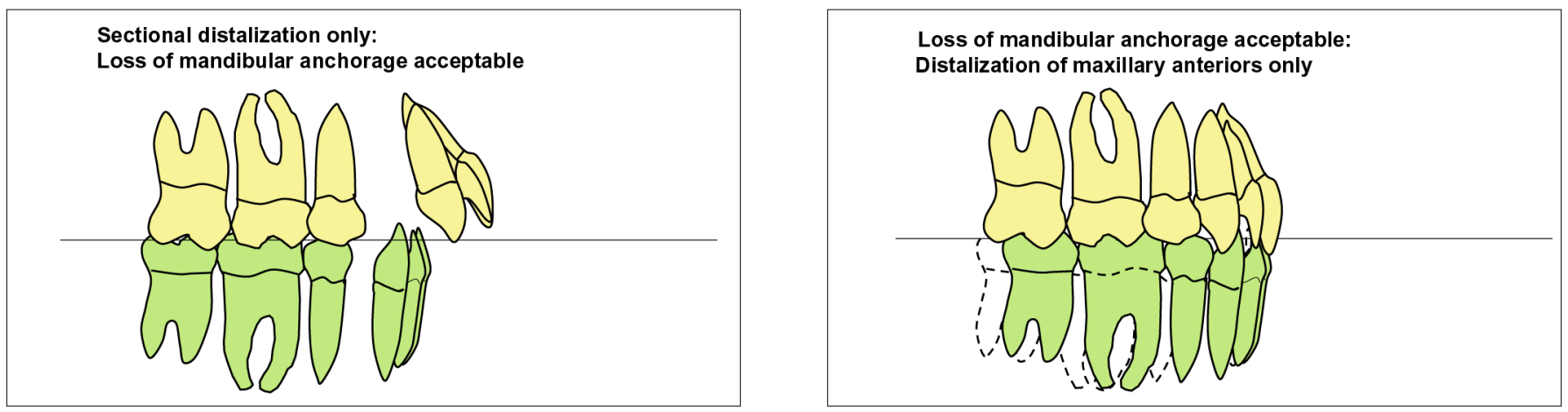

Figures 3 and 4

Only sectional distalization needed, loss of mandibular anchorage possible and desirable.

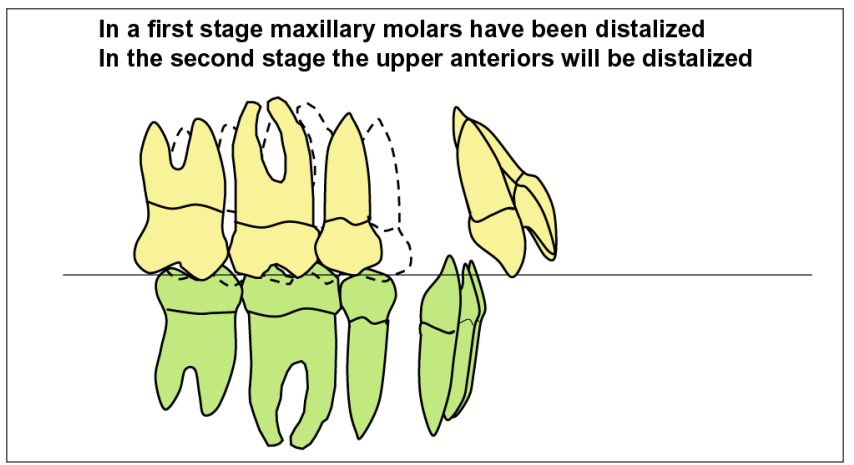

Figure 5

Global retrusion of upper arch in two-stage strategy: 1 st stage sectional posterior distalization and 2 stage anterior distalization. 
force is applied to the teeth being retracted, or,

- direct mechanics where the miniscrew itself is the basic anchorage unit from which force is applied to the teeth being retracted, incisors and/or canines.

Retraction of only the anterior teeth is required when:

- only the anterior teeth need distalization because while they are in a Class II position, the buccal teeth are in a Class I position (fig. 2);

- a loss of mandibular anchorage is possible and desirable (fig. 3 and 4)

- in a second stage procedure the anterior teeth need distalization after the posterior teeth were moved distally in a first stage (fig. 5)

\section{2-1-2-Global movement of the arch}

When the upper arch requires only 1 to $2 \mathrm{~mm}$ of distalization, which is what is needed in forcible anchorage preparation, orthodontists can adopt a strategy of "en masse" arch distalization.

But when the required distal movement is more substantial, a two-stage procedure becomes necessary:

- to accomplish distal movement of the buccal and posterior teeth the anterior teeth are used as the anchorage bloc and miniscrews can reinforce them (fig. 6) in obligatory indirect mechanics;

- for retraction of the anterior teeth the posterior teeth serve as the anchorage unit. Use of the miniscrew is obligatory for the second stage to reinforce posterior anchorage and in retraction of anteriors

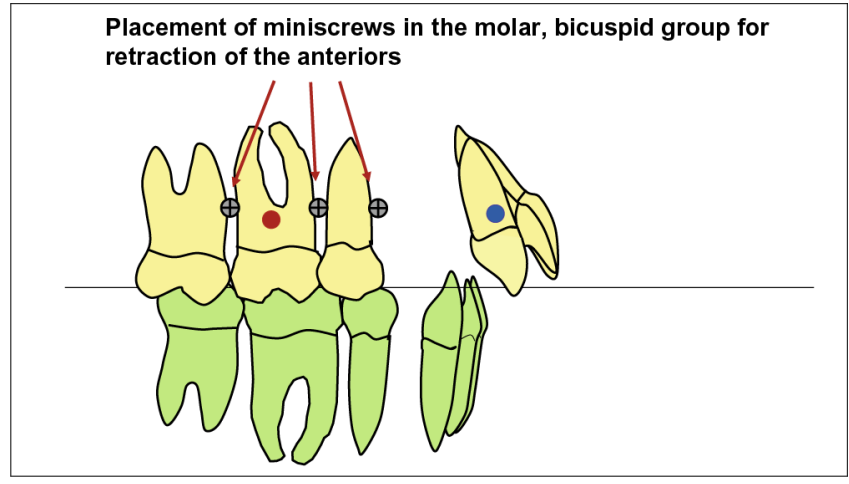

Figure 6

Placement of the miniscrews for retraction of the anteriors.

seamless. Mechanics may be direct or indirect (fig. 7 and 8).

The second stage corresponds precisely to the procedure described in 2 - 1 - 1, one that has been a wellknown and effective orthodontic tactic for generations. But the first step, distalizing molars, poses a question that has perplexed and challenged orthodontists for at least as long. Where can reliable anchorage for distalizing groups of molars be found?

\section{2 - 2 - Retracting the anterior bloc of teeth}

In this procedure the bicuspid and molar teeth serve as anchorage units for retraction of the anterior teeth. Orthodontists have traditionally used anchorage preparation and other schemes to prevent unwanted reciprocal movement of the anchorage teeth but this has not always been possible.

The six anterior teeth can be retracted en masse but to prevent excess strain on the anchorage units 


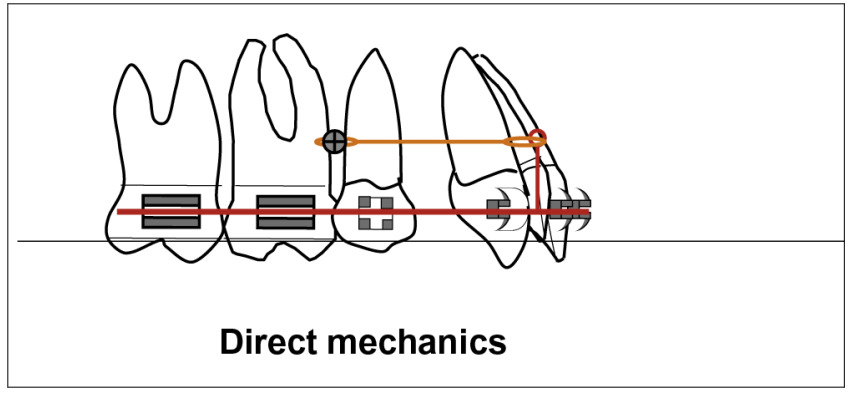

Figure 7

Direct mechanics for retracting anteriors.

this has not always been advisable. Instead orthodontists have moved the canines distally first and the four incisors later, in a second step.

But today miniscrews can be placed within the anchorage bloc (fig. 6) in the interproximal spaces between the first and second bicuspids, between the second bicuspids and the first molars, and between the first and second molars. Relying on the stable anchorage that these miniscrews provide, orthodontists can elect to retract the anterior teeth with a direct or with an indirect technique.

By placing the miniscrews as distally as possible orthodontists can avoid adding a vertical component to retraction of anterior teeth by either the direct or the indirect methods. But if a mechanical analysis shows that a vertical component is desirable, to correct an overbite, for example, the miniscrew should be placed more anteriorly so that force applied to the arch wire from that relatively elevated point will tend to intrude the incisors as they are retracted.

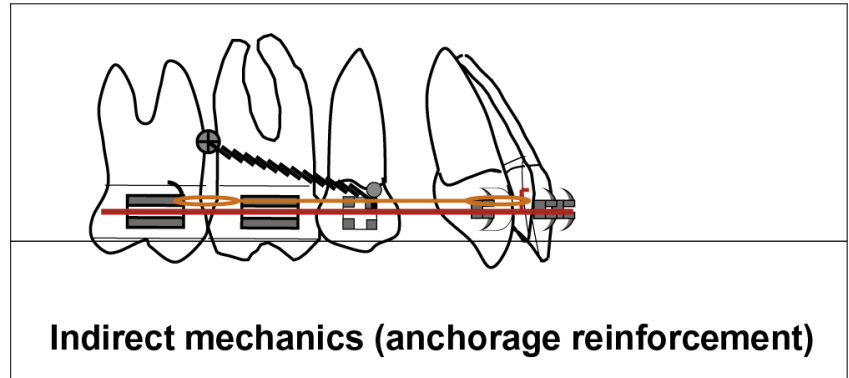

Figure 8

Indirect mechanics for retracting anteriors.

\section{2 - 3- Retraction of the entire arch}

Orthodontists can retract all the teeth in the maxillary arch a short distance of a few millimeters with direct mechanics but a thick septum will limit this type of movement in the anterior sector. Orthodontists, of course, can increase this distal movement by incorporating tip-backs in the set up either by making appropriate bends in the arch wire or by using brackets with pre-adjusted tips. Global movement is enhanced when bilateral edentulous areas exist in the upper arch.

But orthodontists more often determine to conduct upper arch retraction in two stages in the fashion we previously described (fig. 5). There are different possible methods for stage 1 including distalizing premolars and molars with the anterior bloc serving as the anchorage unit.

\section{2 - 3-1 - Cases where molars are extracted}

Miniscrews are especially useful in therapies employing differential molar 
extractions or as previously suggested for severe Class II malocclusions in Merrifield's guidelines.

When the third molars have already erupted or are clearly in a position to erupt successfully, the orthodontist may decide to have upper first molars or upper second molars removed to facilitate distal movement of the teeth anterior to them with the treatment envisaging the third molars acting eventually as substitutes for the extracted teeth. This greatly shortens the duration of treatment especially if miniscrews have been placed to support anchorage.

The amount of dental tooth material lost with this procedure is easy to measure. When maxillary first molars are extracted, the upper molar group will consist of second and third molars instead of first and second molars. The loss of occlusal surface, accordingly, will be equal to the anatomic size difference between first and third molars. If the measured difference is $20 \%$, the effective loss of biting surface will be $10 \%$.

\section{- Extractions of upper first molars}

When upper first molars are extracted, mechano-therapy does not have to move any molar teeth at all, but it must block any forward movement of the upper second molars into the space.

Orthodontists can accomplish this by inserting a rectangular steel arch wire with spurs soldered mesial to the second molars or with a step or bayonet bend incorporated into the arch wire at the same points and ligating the wire to the anterior miniscrew. Then the first and second premolars can be distalized rapidly with elastic chains ligated to the second molars supplemented by open coil springs compressed between the first bicuspids and the canines (fig. 9 and 10).

\section{- Extractions of upper second molars}

Orthodontists can readily distalize single molar teeth with simple mechanics using all the premolars and canines supported by a miniscrew placed in the interproximal space between the cuspid and the first bicuspid and ligated to the cuspid as anchorage and then positioning a coil spring compressed between the second bicuspid and the first molar (fig. 11). If there are no spaces between the canines and the bicuspids, no further adjustment to the arch is required but if there are spaces the orthodontist can preserve them by inserting closed coil springs on the arch wire that will block mesial slippage.

When the molars have been retracted the two bicuspids on each side can be distalized in tandem by a coil spring compressed against the first bicuspid, the first molar having been blocked against mesial slippage by a step in the arch wire.

\section{2 - 3-2 - Cases with no molar extractions}

In this case the second molars, the first molars, and the second bicuspids were distalized individually, one at a time (fig. 12 and 13) with indirect mechanics.

The anchorage was first provided by the anterior bloc of teeth plus canines and bicuspids ligated together and from the canine bracket to the miniscrew placed interproximally between 

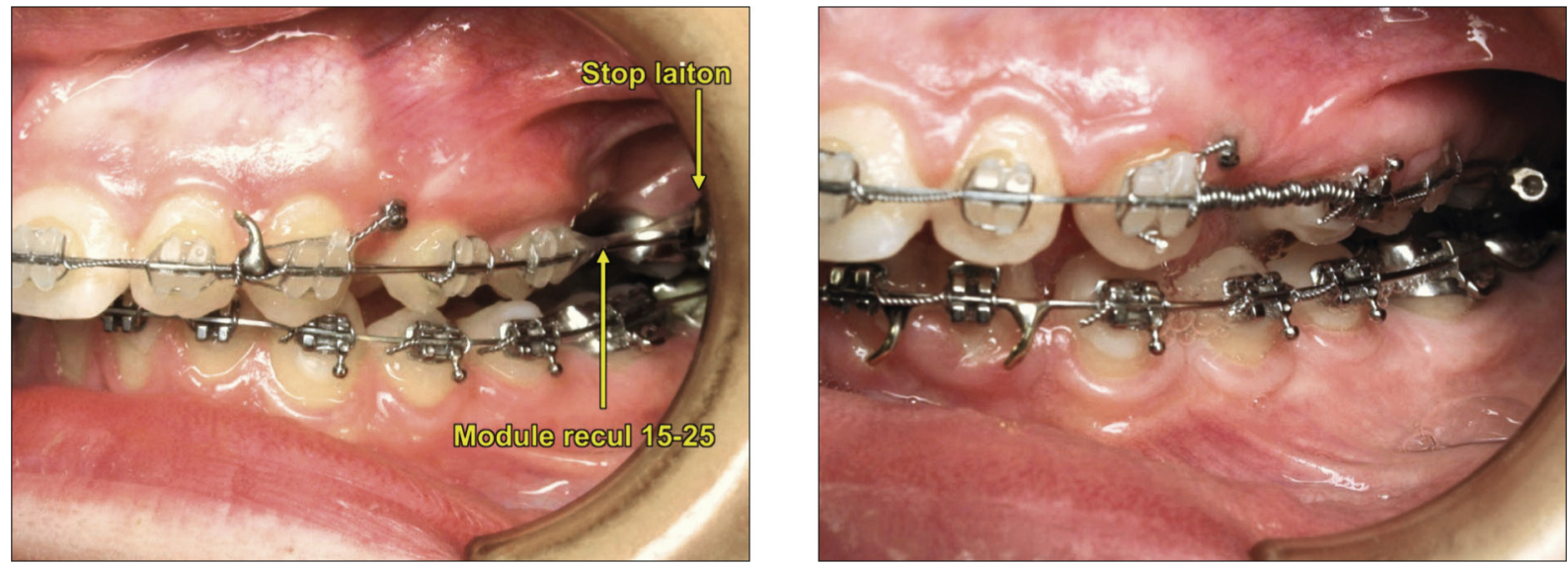

Figures 9 and 10

Extraction of first molars: distalization of second premolars while second molars were blocked from moving mesially by archwire stops. The second molars, canines, lateral incisors, and central incisors plus the miniscrew ligated to the archwire provide anchorage. The first and second bicuspids are distalized simultaneously, first by elastic chains attached to the second molars and finally by coils compressed between the canines and the first bicuspids (fig. 10).

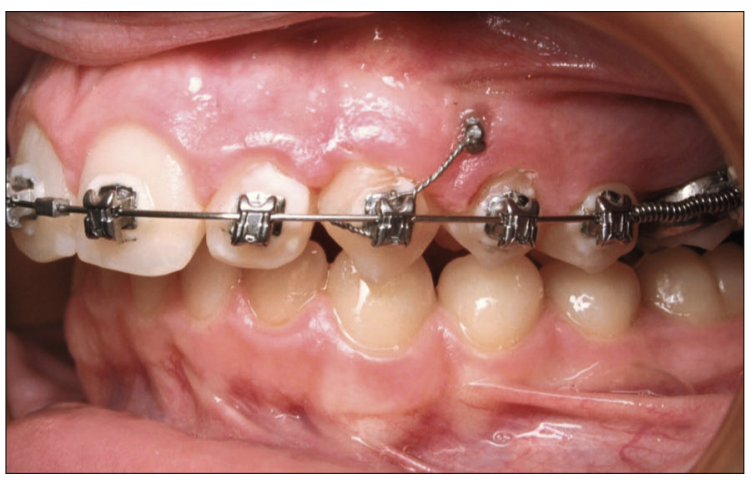

Figure 11

The first molars and then the premolars are distalized in a case where second molars were extracted. The first molars are moved distally by a coil spring compressed between the first molars and the second bicuspids, in traction anchored by the centrals, laterals, canines, and first and second premolars supported by the miniscrew.

the canine and the second bicuspid (the first bicuspids had been extracted years before). The second molars were distalized by a coil spring compressed between the second bicuspid and the second molar, sliding over the first molar that was not yet banded. Spaces between canines and second premolars were preserved by coil springs. Only the mesial wing of the premolar twin bracket is ligated, which facilitates placement of the coil spring and counterbalances the tendency of that tooth to rotate mesio-buccally. After movement of the second molars has been completed they are locked in place by arch wire stops and bands with tubes are cemented to the first molars. Now the upper anchorage group consists of second molars, second bicuspids, canines, and lateral and central incisors and anchorage preparation in the lower arch will be accomplished with Class III mechanics (fig. 13).

Depending upon overall anchorage requirements and clinical exigencies associated with distalization of maxillary premolars and molars, this can:

- when distalization of upper anterior teeth can be accomplished with 

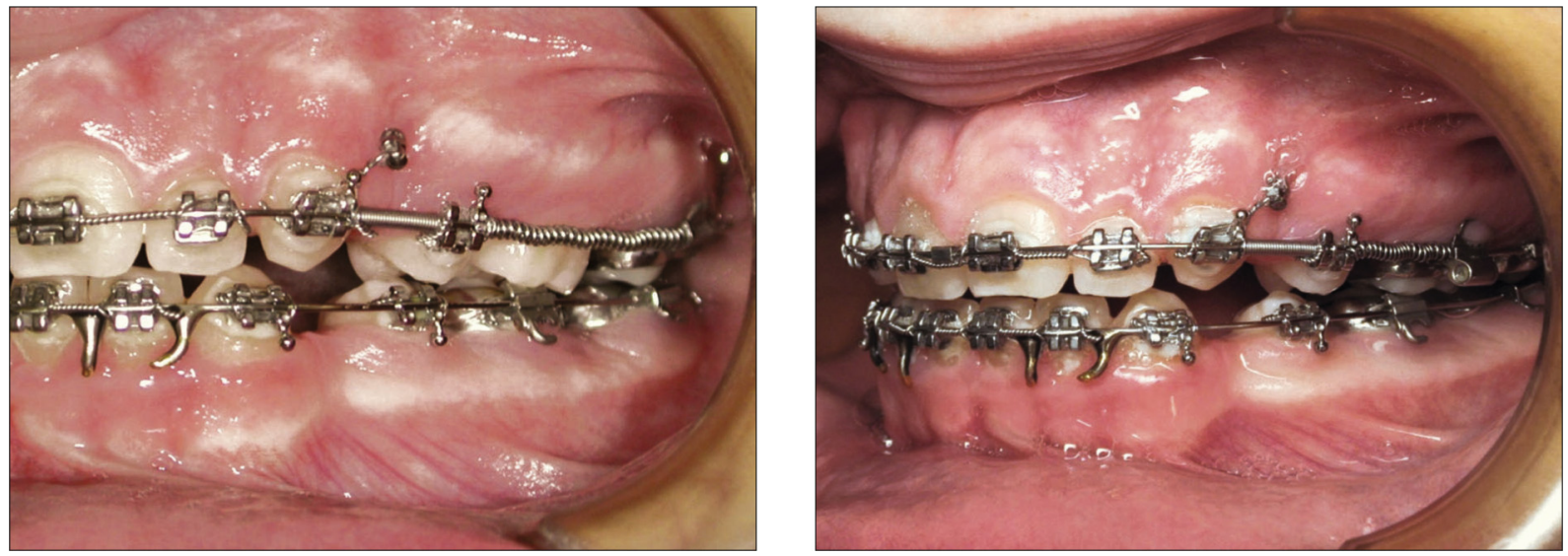

Figures 12 and 13

Complete distalization of second molars, first molars and second bicuspids.

The spaces left by this patient's previous loss of upper first premolars had long since closed up. The distalization of the second molars was accomplished by a coil spring compressed between that tooth and the second premolars that slid over the unbanded first molar (fig. 12). The maxillary incisor, canine, and second bicuspid teeth together with the miniscrew make up the anchorage unit. Note how the upper first molar had spontaneously followed the second molar in its distal movement. When the second molars were in place, the first molars were banded and moved distally in their turn by a coil springs compressed against the second bicuspids, while the second molars were held in place by a stop pinched into the archwire (fig. 13). Now the anchorage unit consists of the maxillary incisors, canines, second bicuspids, second molars, and the miniscrew. Note how the spaces between the cuspids and second bicuspids were maintained with closed coil springs. Class III elastics helped prepare anchorage in the lower arch and made the use of mandibular miniscrews unnecessary.

reciprocal anchorage, avoid the need for mandibular miniscrews;

- eliminate the need for posterior maxillary miniscrews for retraction of the upper anterior teeth when this can be accomplished either with reciprocal anchorage on the over-corrected upper molar teeth or with Class II mechanics.

In every case of global distalization of the maxillary arch, after the most difficult phase - the distalization of the posterior teeth that we have just described - the anterior teeth must be moved distally, either with reciprocal anchorage if it had been possible to over-correct the occlusal relationships of the premolars and molars in the first phase, or by placing miniscrews posteriorly as described in section $2-2$.

\section{3 - CLINICAL AND TECHNICAL MODALITIES}

Orthodontists have to make practical choices about the best sites for placing miniscrews, the modalities of tooth-borne anchorage and of the stable anchorage supplied by miniscrews, the force modules, the man- 
agement of anchorage, and the management of the successive distalization of the molars and the premolars.

\section{3 - 1 - Position of the miniscrew}

For antero-posterior discrepancies we primarily select interproximal areas for miniscrew sites. We base this choice on anatomic and mechanical considerations.

\section{- The anatomic}

- Amount of available bone interproximally. If a desirable site is not quite adequate it may be possible to improve it by opening space or improving the axial inclination of the roots of the proximal teeth,

- Quality of the gingiva,

- Conditions of surgical access,

- Conditions of mechanical access, taking into account the difficulties of access for appliance components like steel or elastic ligatures, particularly in direct mechanics where ligatures or other attachments must be changed.

- State of healing. Extraction sites where surgeons had to perform a considerable amount of bone manipulation unless the area has had three to six months to heal.

\section{- The mechanical}

In using indirect mechanics to distalize the posterior teeth with the anterior sector serving as anchorage, the miniscrew should be placed as far as possible from the teeth to be moved so the orthodontist can include as many dental units as possible in that group. Miniscrews placed interproximally between the upper first and second molars can serve as anchorage only for distalization of those second molars but miniscrews placed in the space between the canine and the first bicuspid can assist in distalization of all the premolars and all the molars. After buccal teeth have been moved into the desire position, orthodontists can often retract the anterior teeth reciprocally; in which case the spaces between canines and first bicuspids become the preferred sites.

In moving a sector of teeth in a single arch, retracting the upper anterior teeth, for example, the miniscrew is placed amid or just mesial to the posterior anchorage unit.

With an anchorage group made up of second bicuspids and first and second molars, the miniscrews can be inserted interproximally between second bicuspids and first molars, between first and second molars, or, for direct mechanics only, just mesial to the second bicuspids.

- If orthodontists wish to add a vertical component to the anteroposterior traction, they should place the miniscrews as close as possible to the teeth being moved. We prefer to insert them just mesial to the second bicuspids in retracting the anterior bloc.

- But if no vertical component is desired, orthodontists should place the miniscrews as far posteriorly as possible. Thus, in indirect mechanics, the ligature ligating the miniscrew just distal to the second bicuspid directly to the second bicuspid or to the archwire will be almost parallel and minimize vertical traction.

\section{3 - 2 - Modules of force}

The modules employed are the same as those used in conventional mechanics: 
- modules that are activated by opening such as Bull loops or coil springs that are opened to supply traction when they are compressed;

- modules that supply traction by closing such as Bull and key loops, elastic ligature power chains, and helical compression springs.

\section{3 - 3 - Anchorage modalities joined to a stabile element}

Orthodontists join a miniscrew to dental anchorage units with twisted metal ligatures tied either to a bonded tooth bracket or to a hook on the archwire.

\section{- Ligation to a bracket}

The orthodontist affixes the ligature to the bracket either by tying it to a hook soldered to the bracket or by threading it through the mesh at the base of the bracket and under the archwire, which is the simplest solution. When the miniscrew is thus added to the anchorage orthodontists can then undertake any conventional intra-arch mechanics such as arch with loops or canine retraction arch

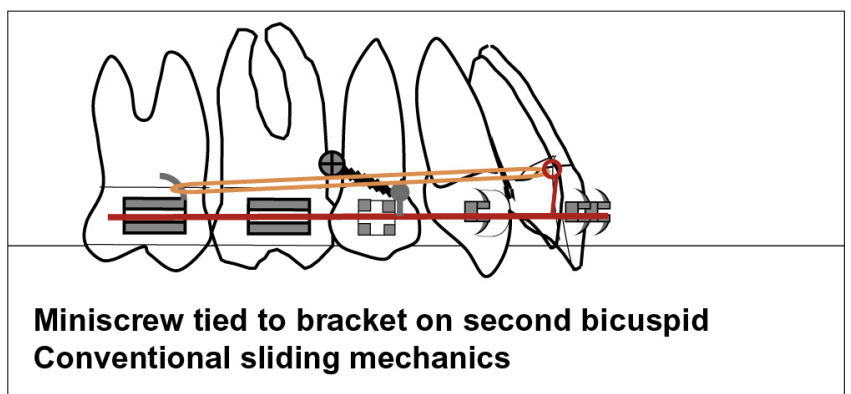

Figure 14

Indirect mechanics, anchorage provided by miniscrew tied to second bicuspid bracket and unit of second bicuspids, and first and second molars for conventional sliding mechanics. without fearing interference from the ligature wire, which is attached, once and for all, under the archwire to a bracket of one of the anchorage teeth, usually the second bicuspid, but they must remain vigilant. Only this tooth is blocked and the mechanics must be based on it. The archwire itself is not blocked (fig. 14). If a problem develops around the miniscrew, threatening its integrity, the tooth ligated to it will be displaced iatrogenically.

\section{- Ligation to an archwire}

The miniscrew can be ligated to a brass hook soldered to the archwire in which case the orthodontist will have to alter mechanics because only the archwire will be blocked: intermediate teeth will be free to slide. Orthodontists can block a tooth with a spur soldered to the archwire or with loops, bayonet bends, or steps bent into the archwire (fig. 15).

\section{3 - 4 - Anchorage modalities applied to teeth being retracted}

Let us take the example of retraction of an anterior bloc of teeth.

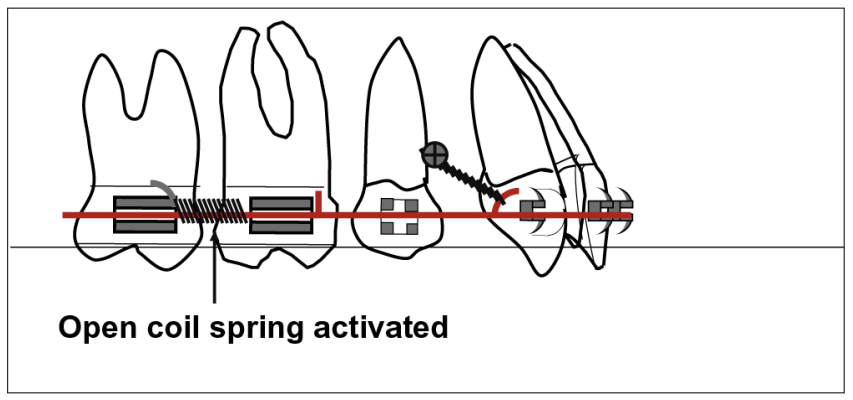

Figure 15

Indirect mechanics, anchorage provided by miniscrew ligated to archwire hook distal to canine. Posterior units are distalized by a compressed open coil. The important problem of maintaining existing spaces is managed by placement of closed coil springs. 
Anchorage is usually derived directly from the miniscrew. But the anchorage varies depending on the whether traction is being applied to a single tooth or a group of teeth, how the force is being applied, and, finally, on the type of global mechanics being employed.

For moving a single anterior tooth, it is preferable to use elastic ligature power chains or a spring acting directly on the tooth's bracket rather than a sliding hook. For retraction of a group of teeth, incisors, for example, orthodontists usually prefer applying force to the archwire through omega bends, pinched or soldered hooks, which have the advantage of allowing for adjustments of the vertical component of the force application by regulating the respective heights of the miniscrews and the site of attachment on the archwire.

For retracting premolars and molars the miniscrews may be ligated to a sliding hook on the archwire that compresses an open spring pushing against the posterior tooth (fig. 16).

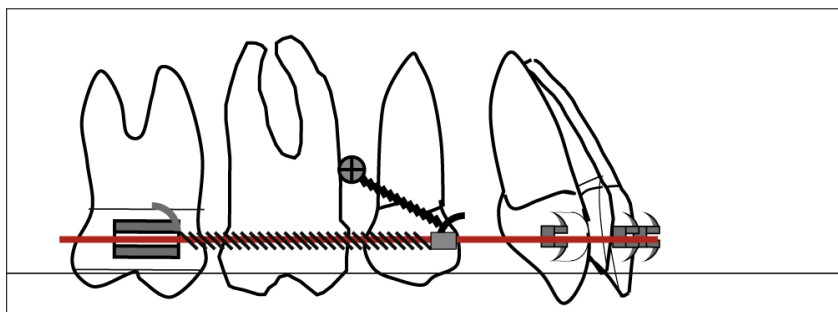

Open coil spring being activated

Figure 16

Appliance conformation where force is applied from the miniscrew, which is attached to a sliding hook on the archwire that compresses the open coil spring against the second molar being distalized. No anteroposterior force is exerted on the arch itself.

\section{3 - 5 - Managing anchorage}

\section{3-5-1-Definition and limitation of anchorage units and teeth being moved}

- The anchorage unit should be as strong as possible, incorporating as many teeth as possible

In direct mechanics, the anchorage group, by reason of the number of its dental units, assures transverse and vertical control of the arch.

In indirect mechanics the union of the dental anchorage teeth to the miniscrew by means of a metal ligature is a ball joint that allows for the displacement of the anchorage force on the arch wire from the miniscrew with a range equivalent to the length of the twisted metal ligature. The length of tooth movement is decreased but the stability of anchorage is greater.

- The number of teeth being moved should be low in order to preserve the efficiency of the sliding process.

For example, it is better to retract the canines first and the four incisors later in a second stage than it is to attempt to move all six teeth at once, "en masse".

\section{3 - 5- 2 - Stable anchorage groups at a distance}

The necessity of having a favorable anchorage relationship between the stable unit and the teeth to be moved, even with the support of the miniscrew, inclines orthodontists to use the conventional "groups of teeth at a distance".

In the retraction of the premolars and molars stage with indirect 
mechanics and, at first, anchorage supplied by the canine-incisor bloc, the second molars as soon as they been placed in their new positions are blocked with a stop of a spur or an archwire bend and added to the anchorage group. The process is repeated with the first molars.

\section{$3-5-3$ - Reversal of the anchorage and retraction sectors}

In distalizing the entire maxillary arch in severe Class II cases, for example, orthodontists follow this procedure:

- $\mathbf{1}^{\text {st }}$ stage: obligatory indirect mechanics: retraction of premolars and molars with anchorage supplied by anterior bloc.

Placement of a miniscrew is obligatory for the second stage.

- $\mathbf{2}^{\text {nd }}$ stage: either direct or indirect mechanics: retraction of the canineincisor bloc with anchorage provided by the posterior teeth, which involves a reversal of the anchorage and retraction sectors. Increasing the number of units in the anchorage group is favorable for the mechanics of this stage but unfavorable for the following stage and vice versa.

\section{3-6-Management of the successive retraction of the molars and the premolars}

To successfully manage the successive retraction of the posterior teeth orthodontists must overcome certain delicate problems:
- the risk of axial rotation of molar teeth;

- the difficulty of inserting expansion springs in the interproximal spaces that are small at the outset;

- the risk of loss of existing anterior spaces owing to the mesial drift of the teeth supporting the open coil springs;

- the tendency of distalized posterior teeth to relapse mesially and the need to block them efficiently so that they can eventually be utilized as anchorage for the retraction of other teeth or for anchorage preparation in the mandible.

\section{$3-6-1-$ Risk of the loss of control against axial rotation of molars}

The strength of the spreading force of the springs tends to rotate premolars or molars anterior to the open coil spring mesio-buccally and the premolars or molars posterior to it distobuccally during the course of the retraction action.

The second molar, always furnished with a tube, moves distally without any tendency to rotate any further than the measurable and relatively insignificant play of the archwire in the tube will allow.

The first molar, however, tends to rotate mesiobuccally as the second molar is retracted and then distobuccally is it is itself retracted. We have found three solutions for this problem.

\section{- When the upper first molar has} an active self-ligating bracket

To retract the upper second molars we compress an open coil spring between the first and second molars, 


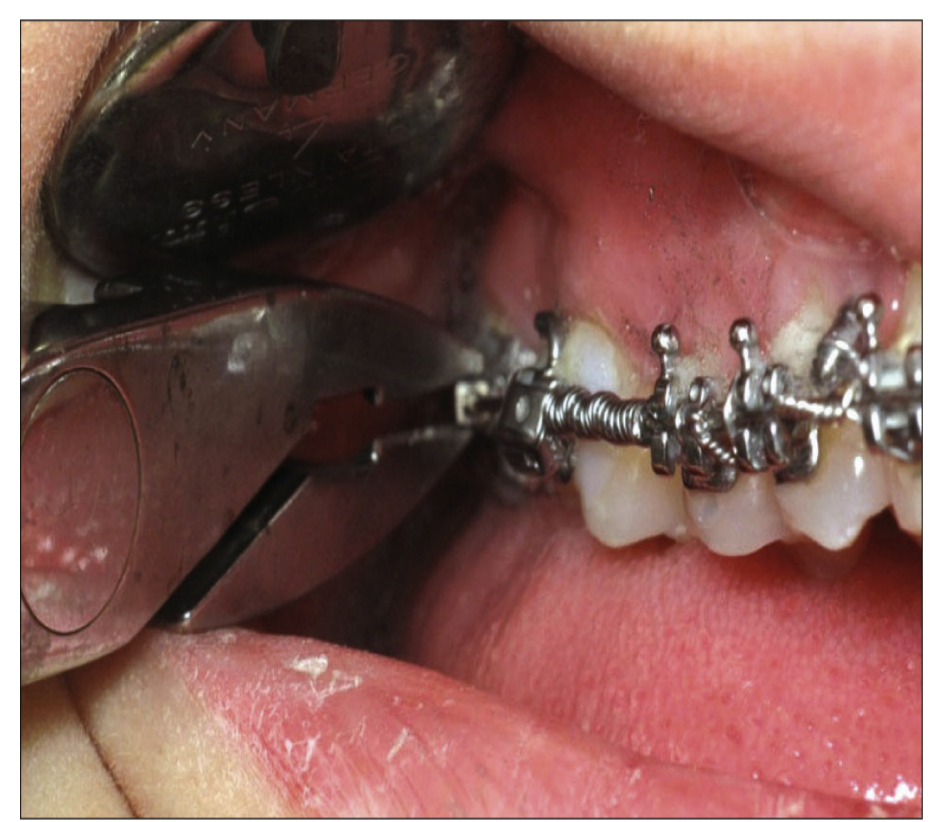

which is compressed again to lock the first molar in place after its retraction, during which the second molars are blocked by arch wire bends. There is no need to bypass the tube on the upper first molar. It is simply left open. When all the molar teeth are retracted, the compressed spring is placed between the upper second bicuspid and the upper first molar whose lock is closed again (fig. 17).

- When the upper first molar has a tube that may or may not be convertible into a bracket but which, in any case, must not be "decapsulated."

Placing a compressed coil spring between the tubes of the upper first and second molars is practically impossible so we remove the first molar band place the compressed open coil spring between the second bicuspid and the second molar (fig. 12) and then re-cement the molar band with a tube and put the compressed coil spring between the second premolar
Figure 17

Complete distalization of the premolars and molars in a non-extraction case: starting with the progressive distalization of the second molars and then the first molars. We first distalize the second molars with compressed open coil springs that we are able to position readily between the self-ligating brackets on the first molars and the second molar tubes. When the second molars are in place we can then readily place springs between the self-ligating first molar bracket and the second bicuspid bracket. Note that we have blocked the distalized second molars by making deformations in the archwire and the molar tube. But now blocking the second molar can only be accomplished with a pinched stop on the arch wire, the tube on the first molar making a bent arch wire or soldered stop impossible.

\section{- When the upper first molar has a} conventional bracket or a convertible one that we "uncover" it is impossible to install a strongly tightened ligature using only the mesial wings of the brackets during the retraction of the upper second molars or the distal wings when retracting the first molar itself. This is especially true for orthodontic offices that use convertible brackets with an extra-oral force tube on first molars. So this isn't a solution that we recommend.

The control of axial rotations of premolars, whether they occur when they are being retracted or are serving as anchorage units, does not pose a serious problem because they are usually furnished with twin brackets 
so that anti-rotational ligature ties may be made on both arms of one wing alone.

\section{$3-6-2$ - Difficulty in inserting coil springs}

This spring is tied to the attachments on the premolars and the molars.

The traditional way of removing interference from an intervening tooth is to remove its band while the tooth distal to it is being retracted, for example the first molar band is removed when the second molar is being distalized by pressure exerted against the second premolar, or the second bicuspid is de-banded while the first molar is being distalized by pressure exerted against the first bicuspid (fig. 12).

To introduce a coil spring, even if it has been pre-compressed, between tubes on the first and second molars on an arch wire already in place the orthodontist must perform acrobatics.

On the other hand orthodontists can easily introduce a coil spring if the anchor tooth is equipped with a twin bracket that has four wings or a selfligating bracket.

\section{3 - 6-3 - Risk of loss of existing anterior spaces from mesial drift of a tooth anterior to the active spring}

In extraction cases there are spaces throughout the arch particularly in the premolar-canine group. During second molar distalization, when an open coil spring is compressed between that tooth and the first molar, closure of undesired spaces between canines and first bicuspids, first bicuspids and second bicuspids, and second bicuspids and first molars occurs automatically with mesial movement of teeth distal to the spaces in spite of the miniscrew being anchored to the canine. This effect continues with the successive distalization of the first molars, second bicuspids, and first bicuspids.

But orthodontists must be careful to preserve desirable spaces by placing stops in the archwire mesial to the anterior tooth serving as anchorage for the compressed spring, as shown in figure 15 , or by placing closed coil springs in the space to be saved or by anchoring the open coiled spring directly to the miniscrew with a twisted ligature tied to a sliding hook at its interior end as shown in figure 16.

In distalization of the anterior bloc anchored on the second molars or the first molars or both the posterior teeth will move mesially reciprocally enough to close any residual spaces or those created therapeutically in the molar and premolar regions, the twisted ligature anchoring the first bicuspid to the miniscrew in the interproximal space between first bicuspid and canine having no control over that.

\section{3 - 6-4-Blocking already distalized teeth}

By blocking already distalized teeth orthodontists not only prevent them from slipping mesially but also keep them as useful anchorage units for distalization of anterior teeth or for anchorage preparation in the mandible. 
Accordingly, after the distalization of the second molars, the anchorage group for distalization of the first molars will be all the bicuspid teeth, the canines, all the anterior teeth, and the miniscrew attached to the canine. After distalization of the first molars, we usually retract all four bicuspids simultaneously, using the anteriors, the canines, and the molars as anchorage plus the miniscrews affixed to the canines.

Stops can be screwed, pinched, or made by archwire bends or soldered hooks but to block second molars already distalized only pinched or screwed stops can be used if the first molars are equipped with tubes or transformable brackets that cannot be covered.

We find the best way to prevent mesial slippage of already distalized molars is to bend stops in the arch. It is not a good idea to place a stop only mesial to the second molars and to ligate the first molars to it because it is difficult to make it tight enough.

\section{3 - 7 - Standard protocol}

We use Roth built-in torque $0.022 \times$ 0.028 brackets and $0.019 \times 0.026$ steel archwires for the sliding mechanics we have described.

- When the first molars are furnished with passive self-ligating brackets the protocol is simple:

1. Anchorage with a miniscrew placed interproximally between canine and first bicuspid and tied to the canine with a twisted ligature wire passing under the archwire.

2. Distalization of the upper second molars with coil springs compressed against the first molars.
3. Blockage of the second molars with archwire steps placed mesial to its tubes and placement of coil spring between second bicuspids and first molars. We prepare anchorage in the mandible with Class III elastics.

4. We block both first and second molars with archwire steps against the first molar tubes. We then distalize all four bicuspids simultaneously with compressed coil springs with ligatures tied to the distal wings of the bicuspid brackets.

5. We finish mandibular arch preparation with Class III mechanics.

6. We use power chain elastics from distalized premolar and molar bloc anchorage unit to retract the anterior teeth combined with Class II elastics.

7. Finishing intercuspation details.

- If we are using convertible brackets that we don't uncover:

1. We use anchorage from a miniscrew placed interproximally between canine and first bicuspid tied by a steel ligature passing under the arch to the canine bracket. We remove the first molar band, the tooth having already been leveled.

2. We distalize the second molar with a coil spring compressed between the second molars and the second molars with ligatures tied to the mesial wings of the second bicuspid brackets.

3. We block the distalized second molars with archwire stops pinched just mesial to their tubes and compress coil springs between the first molars and the second bicuspids. Eventually, we remove the attachment on the second bicuspids and place the compressed 
coils between the first molars and the second bicuspids. We ligate the anchor premolar only on the mesial wing of its bracket. The next step is anchorage preparation of the mandible with Class III mechanics.

The remaining procedures, steps 4 to 7 , are identical to those in the previous list.

\section{4 - ANALYSIS OF MECHANICS USED IN MANAGING ASSOCIATED VERTICAL PROBLEMS}

We shall focus on the sliding mechanics that we use exclusively. By rigorously analyzing the mechanics set in motion we can usually control any moderate vertical malformations associated with the primary problem, the antero-posterior discrepancy.

But serious vertical malformations require, of course, individualized control with the use of additional selectively placed miniscrews.

\section{4 - 1 - Direct mechanics of retracting anterior teeth with a sliding arch, with anchorage supplied by all molars and second bicuspids and the force coming from the miniscrew to teeth being moved*}

When we have placed the two groups of teeth in position and splinted them together with a rigid steel archwire, usually $0.019 \times 0.026$ sliding with negligible friction in 0.022 $\times 0.028$ bracket slots, the action

\footnotetext{
*The anchorage calculations refer to the coefficients of Nabbout ${ }^{1,}$ 7, 8 . An anchorage unit corresponds to the anchorage value of the mandibular incisor.
}

achieved can be compared to a drawer sliding in a dresser. Such a model can be studied by separating the applied forces into the components parallel to the sliding or the "compatible" horizontal forces that are going to mobilize the sliding (opening and closing the drawer) and the forces perpendicular to the sliding or the vertical "incompatible" forces that can only displace the entire structure (the dresser itself).

In our sketches (fig. 18 to 22):

- the compatible component Fh puts the liaison "into play" and retracts the anterior bloc of teeth by thrusting against the six-unit resistance unit: a separate action depicted in the figures 18 and 19;

- The force Fv, incompatible with horizontal sliding, confronts the overall resistance of the arch, 15.5 units; it moves the ensemble of the arch slightly upwards as a function of the arch's resistance and with regard to the center of resistance of the entire arch: a separate action depicted in figures 20 and 21.

- The combination of these two actions achieves the situation depicted in figure 222. 

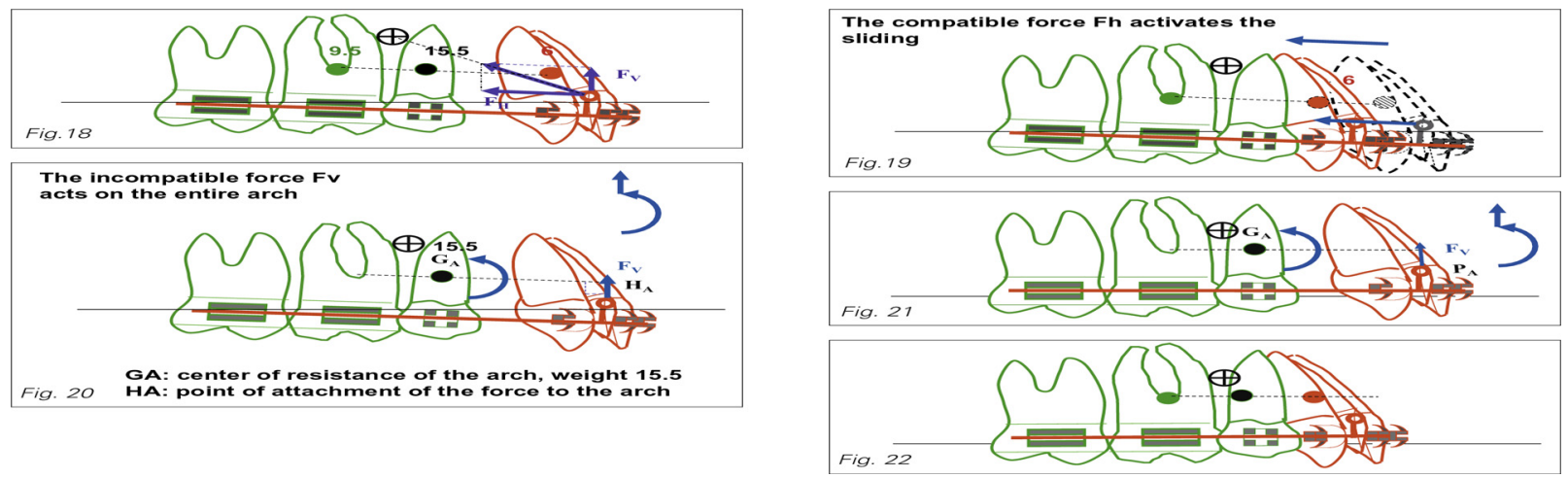

Figures 18 to 22

Sketches of the principle of the direct mechanics of retraction of anterior teeth:

The anchorage weights are respectively 6, 9.5, and 15.5 units for the anterior groups, the posterior group, and the entire arch. The miniscrew located in the interproximal space between the second bicuspid and the first molar carries a module that is attached directly to the anterior group of teeth. The exerted force $F$ can be broken down into:

Fh, a horizontal force compatible with the sliding action and provokes the translation into pure retraction of the anterior teeth, according to the value of the resistance of this group of six teeth

Fv, a vertical force incompatible with the sliding liaison; it confronts the global resistance of the arch, that is displaced globally in accordance with its center of resistance and its anchorage value of 15.5.

Figure 19 illustrates the separate action of the compatible force, Fh.

Figure 20 analyzes the effect that Fv has on the entire arch: intrusion in accordance with the power of the vertical force, Fv and counter-clockwise tilting in conformity to the moment Fv. $G_{A}-H_{A}$.

Figure 21 shows the movement related to the isolated vertical action of force Fv.

Figure 22 shows the global effect of all the F forces, by super-imposing the effects depicted in 19 and 21.

\section{4 - 2 - Indirect mechanics of the retraction of anterior teeth with a sliding arch employing stable anchorage of all the molar teeth and the second bicuspids + a miniscrew ligated to the second bicuspid (the module of force acts from the stable group to the mobile group) (fig. 23 and 24)}

Here again we have two groups of teeth joined by a sliding arch moving in their brackets. The system can be analyzed in the same way we analyzed the direct mechanics system: a sliding element forces an arch movement compatible with a global displacement of a group.

But the stable anchorage unit is tied to the miniscrew by an unbreakable metal ligature: the point of anchorage, the bracket of the second bicuspid, is tied to the miniscrew: it can only move on the spherical surface centered by the miniscrew and the radius equal to the length of the steel ligature. This is an absolute liaison or an "absolute anchorage" that takes precedence over any other liaison. We must, accordingly, analyze the system in giving priority to the absolute liaison. 

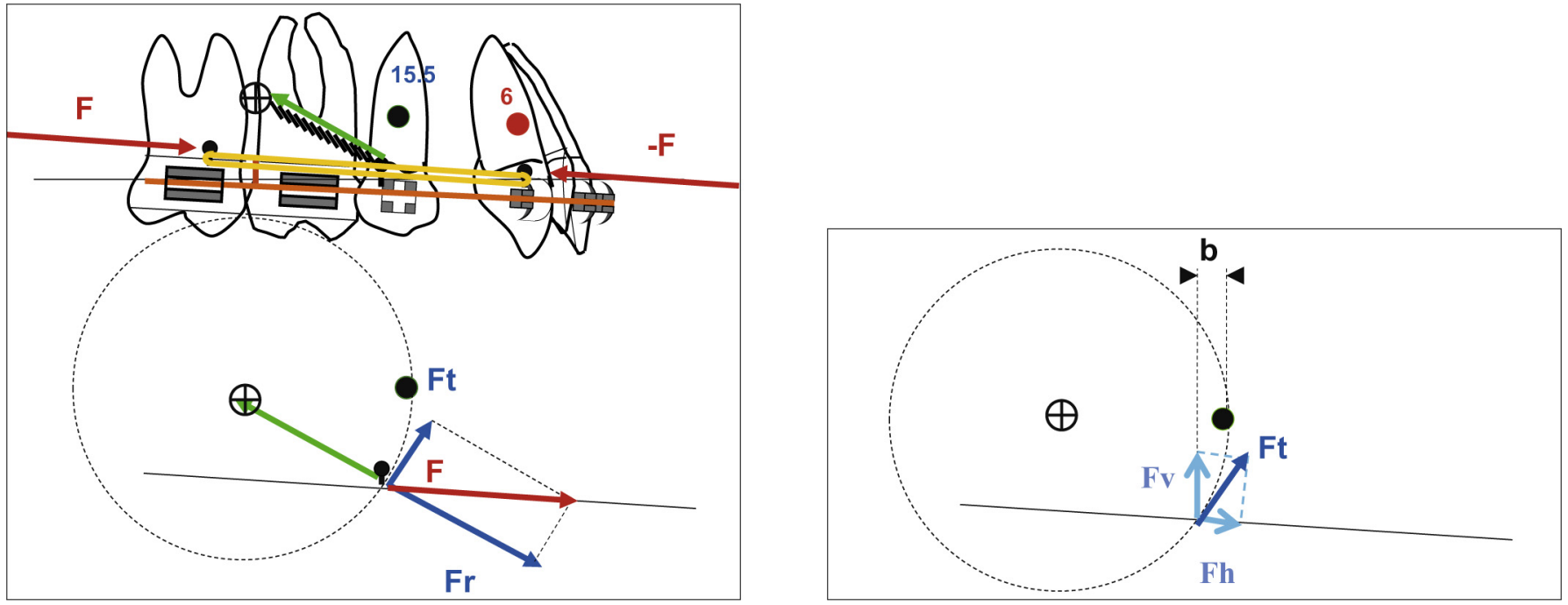

Figures 23 and 24

Sketches of the principle of indirect mechanics for retraction of anteriors.

The stable anchorage groups, made up of 9.5 units, are the same as in the previously described case, but they are reinforced by the fixed miniscrew anchorage attached to the brackets of the second bicuspids.

But this time there are two liaisons:

- a slider,

- an unbreakable metal ligature: the bracket of the second bicuspid can only on the spherical surface centered by the miniscrew and a radius equal to the length of the twisted ligature.

The "absolute anchorage" outweighs the other liaison in strength.

Figure 23 shows the breakdown of the force received by the retraction module at the level of the second bicuspids:

- the radial component Fr is negated by the rigidity of the metal ligature;

- the tangential component Ft can move the bracket on the circle.

Figure 24 delineates the role of Ft.

Its component Fh activates the sliding liaison: thus slightly moving the anchorage unit mesially.

Its component $F_{V}$ is capable of mobilizing the entire arch: depressing it and imparting to it a slight clockwise rotation as a function of the moment b.Fv.

\section{- Absolute liaison}

The drawing together force, $F$ (antero-posterior and parallel to the archwire) exerted on the anchorage unit can be broken down into a tangential force $\mathrm{Ft}$, compatible with the liaison working to displace the second bicuspid bracket on the circumference centered on the miniscrew and an incompatible radial force $\mathrm{Fr}$, that thrusts against the resistance of the metal ligature that negates it.

\section{- Sliding resistance}

Ft can be broken down into a horizontal component that activates the double action sliding, to a very slight mesial amount of the posterior anchorage bloc in accord with its anchorage value of 9.5 units and a vertical component $\mathrm{Fv}$, incompatible 

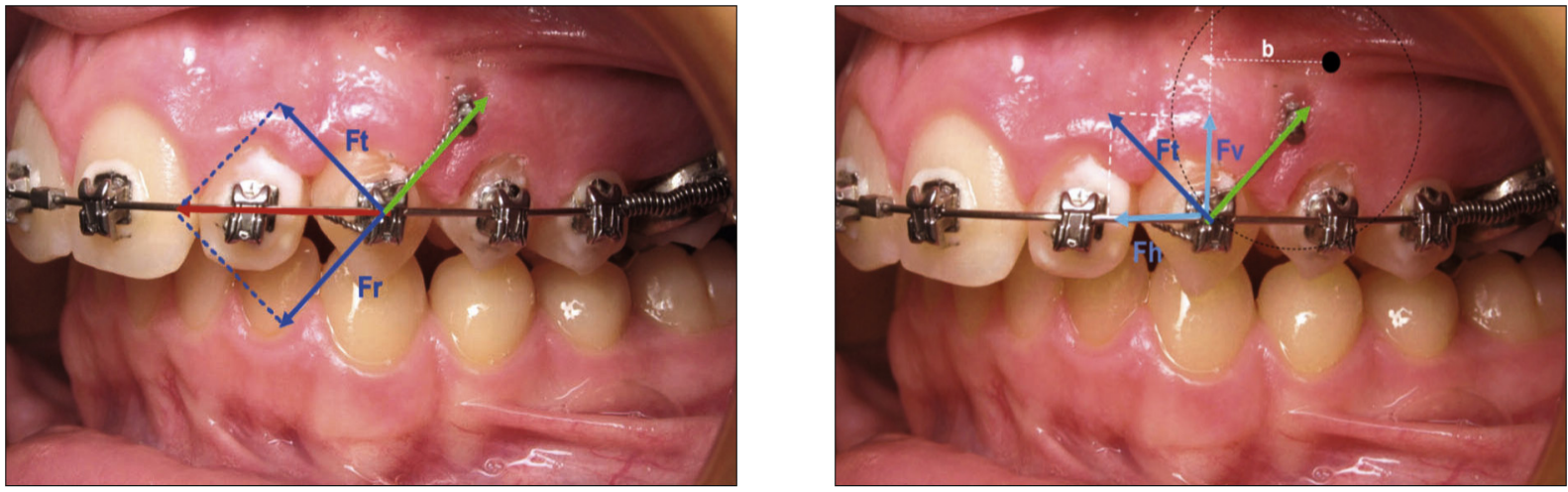

Figures 25 and 26

Mechanical analysis of a case of posterior retraction with indirect mechanics.

The action of the tangential component Ft and the vertical component, strengthened by the length of the lever arm $b$, produced an intrusion and a substantial rotation of the maxillary arch by elevating its anterior sector.
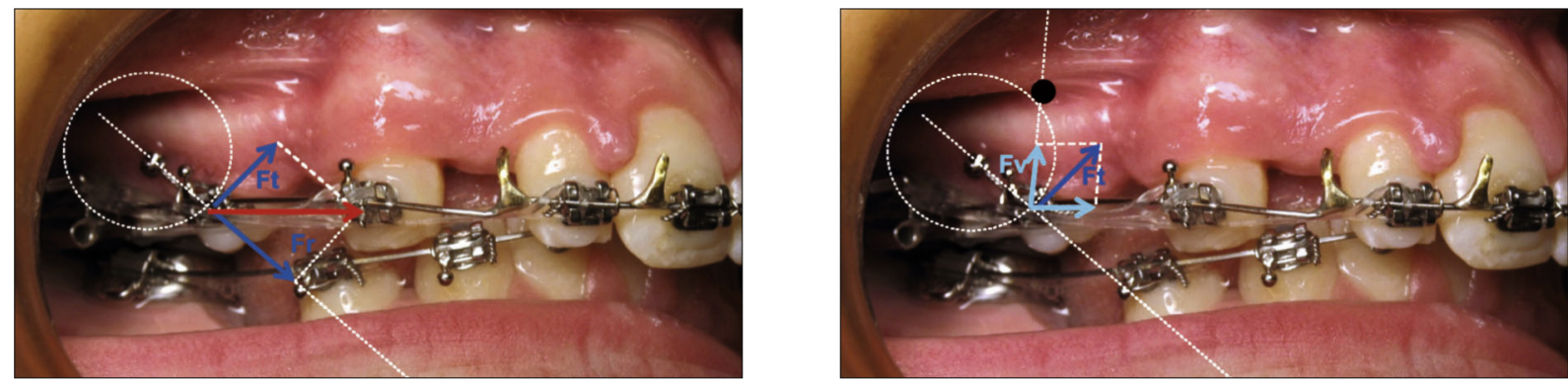

Figures 27 and 28

Mechanical analysis of a case of anterior retraction with indirect mechanics.

In spite of the substantial contribution of the tangential component Ft, and, therefore, of its vertical element Fv, there was no tilting of the arch because of the firm anchorage provided by the miniscrew acting through the second bicuspid bracket, which is located at the arch's center of resistance.

with the sliding, that can displace the entire arch. This arch displacement can be analyzed by taking into account Fv, the arch's center of resistance, and the value of the anchorage, which is 15.5 units. In the case depicted in the sketch, the arch displacement consists of a small amount of intrusion, in accordance with Fv, a clockwise tilting of the arch that is feeble because Fv passes close to the arch's center of resistance. The anterior bloc retracts in accordance with $-\mathrm{F}$, and as a function of the value of its anchorage, 6 units. The sliding unit has a strong retracting action on the anterior bloc (-F and six units) and a small posterior mesializing action (Fh and 9.5 units).

The slight mesial displacement of the anchorage group and the global displacement of the arch produce a displacement of 5 tangent to the circle. 


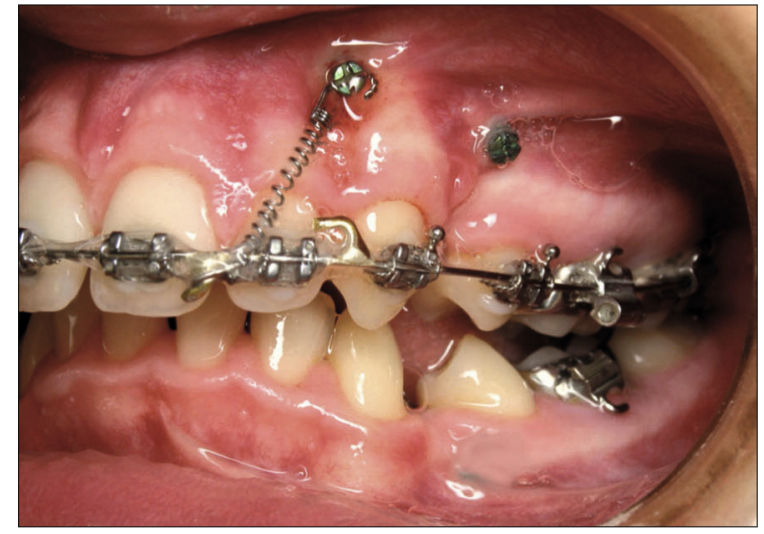

Figure 29

In this case an anterior miniscrew, dedicated to vertical action, was placed between the lateral and canine to help correct an antero-posterior discrepancy with a strong vertical component.

This emphasizes the benefits of basing anchorage on the upper second bicuspids, which, because of their location near the arch's center of resistance, encourage very little tilting of the arch.

Analyses of two clinical cases illustrate this calculation method: the first in figures 25 and 26, where counterclockwise movement was desired, and the second in figures 26 and 27 where there was scarcely any tilting of the arch.

\section{$4-3$ - Control of the vertical dimension}

The analyses that we have just described show that if orthodontists wish to make adjustment in the vertical dimension, of, perhaps, an overbite associated with a clockwise tilting of the maxillary occlusal plane, in the course of Class II treatment, they should employ direct mechanics because this technique can incorporate more different types of adjustments in its Class II therapy. Using it, orthodontists can:

- modify the respective heights of the miniscrew and its attachment to the arch with a long or a short hook, crimped or soldered;

- choose a more anterior site for a highly placed screw thus increasing the vertical component acting from below upward;

- advance the anchorage attachment on the archwire to lengthen the lever arm with regard to the center of resistance of the arch.

With indirect mechanics, on the other hand, the anchorage is located at the second bicuspids, in close proximity to the center of resistance of the arch and orthodontists can reinforce the vertical component $\mathrm{Fv}$ to, at most, the value of $F / 2$ by elevating the position of the miniscrew as much as possible, which also reduces the antero-posterior control they have of the stable anchorage unit.

If orthodontists require substantial vertical control, they must add to the miniscrews deployed for antero-posterior movement other miniscrews placed advantageously for vertical action, which would be above the bloc of anterior teeth as depicted in figure 29. 


\section{CONCLUSION}

The introduction of miniscrews into the toolbox available to orthodontists has, in the first place, made it possible for them to bring standard treatments to a successful conclusion with far less cooperation from patients than was formerly required, but to do this they must employ mechanics that it would be fallacious to consider as simplified. In addition, miniscrews are also probably going to extend the range of malocclusions that they can correctly treat without the aid of extractions or surgical interventions.

\section{REFERENCES}

1. Faure J, Nabbout F, MarshalSixou C, Oueiss A. Centres de résistance des groupes dentaires et mécanique orthodontique. II. Applications. International Orthod 2008;1:3-22.

2. Kim SH, Yoon HG, Choi YS, Hwang EH, Kook YA, Nelson G. Evaluation of interdental space of the maxillary posterior area for orthodontic miniimplants with cone beam computed tomography. Am J Orthod Dentofacial Orthop 2009;135:63541.

3. Kim HJ, Yun HS, Park HD, Kim DH, Park YC. Soft tissue and cortical-bone thickness at orthodontic implant sites. Am J Orthod Dentofacial Orthop. 2006;130:177-182.

4. Lee JS. Applications cliniques des mini-implants en Orthodontie. Traduction Katabi S. et Liger F. Quintessence International.

5. Ludwig B. Miniimplants in orthodontics. Innovation. Anchorage. Concepts. Quintessence International.

6. Nabbout F, Faure J, Baron P, Braga J, Treil J. L'ancrage dentaire en orthodontie : les données du scanner Tooth anchorage in orthodontics: scanner generated data. International Orthodontics, 2004;2:241-256.

7. Nabbout F, Faure J, Braga J. Treil J, Baron P. Anatomie dentaire et orthodontie. L'apport du scanner 3D. Dental anatomy and orthodontics. The benefit of the 3D scanner. Rev Orthop Dento Faciale 2003;37(3):59-73.

8. Nabbout $F$, Faure J, Baron P, Braga J,Treil J. Centres de résistance des groupes dentaires et mécanique orthodontique. I Détermination des centres de résistance. Centers of resistance of tooth segments and orthodontic mechanic. International Orthod 2007;5:437-446.

9. Park HS. An anatomical study using CT image for the implantation of microimplants. Korea J Orthod 2002;32:435-441.

10. Sung J, Kyung $H$, Bae S, Park H, Kwon O, Mc Namara JA. Microimplants in orthodontics. Department of Orthodontics. School of Dentistry. Kyungpook National University Daegu Korea, Ed Dentos, 2006. 